\title{
Change of Sex Gaps in Total and Cause-Specific Mortality Over the Life Span in the United States
}

\author{
YANG YANG, PHD \\ Lineberger Comprehensive Cancer Center, Department of Sociology, and Carolina Population \\ Center, University of North Carolina at Chapel Hill, Chapel Hill, NC \\ MICHAEL KOZLOSKI, MS \\ Center on Aging, Department of Obstetrics and Gynecology, and Department of Sociology, \\ University of Chicago, Chicago, IL
}

\begin{abstract}
PURPOSE-Previous research has led to the expectation that the gap in mortality between sexes narrows in older ages as sex differences in fecundity decrease. However, the patterns and explanations of variations in sex disparities in mortality across the life span and underlying causes of death are not well understood. We conducted a population-based study to further test this hypothesis.
\end{abstract}

METHODS—By using a nationally representative sample of adults $(N=25,254)$ with mortality follow-ups for 18 years, we modeled age variations in sex differences in risks of mortality from leading causes of death.

RESULTS-Male excesses in mortality decrease at older ages significantly for some but not all causes. Differential exposures to social, physiological, and morbidity risk factors account for the late life reductions of the sex mortality gaps completely in circulatory diseases, partially or minimally in the other causes of death. Social status and relationship are more important risk factors for mortality in younger ages, health behaviors are significant for all ages, and physiological dysregulation is more predictive of mortality in older ages.

CONCLUSIONS-Sex differences in the risk of mortality have strong age variations and are cause specific. Additional studies of age acceleration of cancer mortality risk are needed.

\section{Keywords}

Biological Aging; Mortality; Physiological Processes; Postmenopause; Sex Differences; Social Behavior

\section{INTRODUCTION}

Sex differences in mortality have intrigued, and continue to intrigue, scientists from many disciplines because they arguably provide one of the most promising areas of scientific research into aging and longevity $(1,2)$. Survival advantages in the female have been widely observed across time, space, and species $(3,4)$, but a full understanding of the origins and mechanisms in these disparities is still lacking (5). One glaring omission in the assessment of sex phenotypic differences is how they unfold with individual aging (6). There is 
evidence from demographic data that the pronounced survival disadvantage in males during young adulthood decreases at older ages $(7,8)$ and that cardiovascular diseases account for the majority of the sex gap in adult mortality and the decrease of this gap at older ages ( 9 , $10)$.

Our knowledge is far from complete, however, on the patterns and explanations of variations in this gap. Evidence of a late life reduction in the sex gap is restricted to historical periods up to the 1980s with limited age ranges and causes of death. It is unknown whether such patterns hold in the contemporary U.S. population subject to a different epidemiologic environment. To the extent that age and cause of death provide crucial information on physiological and behavioral pathways through which sex-specific mortality occurs, the age variations in sex differentials in cause-specific mortality can be highly informative to the understanding of the sex difference in longevity and merits further investigations.

The timing of the change in the sex mortality gap brings theories of evolutionary biology of aging and reproductive biology to bear on the physiological mechanisms underlying the change. The converging sex gap can be expected as a result of sex differences in the age pattern of fecundity decrease $(11,12)$ and an accelerated decline of physiological functions in other major regulatory systems after the exhaustion of reproductive potential in women but not men (7). Furthermore, the female sex hormone, estrogen, may protect women at the cellular level in terms of immune competence, fat and glucose metabolism, and cardiovascular compliance (13-17), all major physiological risk factors of mortality $(2,18$, 19). This estrogenic hypothesis implies the loss of physiological advantages caused by the reduction of estrogen after menopause (20).

A separate and sizeable body of demographic and social epidemiologic literature has identified powerful processes by which social status and socially learned lifestyles and behaviors affect human mortality and gender differences therein (21-23). Changes in exposures to social risk factors of mortality in middle to old ages consequently may contribute to age variations in gender gaps in mortality. Convergences in gender gaps can be expected from the age-as-leveler hypothesis that aging narrows and levels social disparities in health by equalizing social statuses and resources in late life $(24,25)$. For instance, eligibility for social welfare benefits such as Medicare, retirement from the paid labor force, and increasing stressful life events such as widowhood and loss of friends in old age can minimize gender differences in survival. Because social conditions have rarely been modeled in relation to age changes in the sex mortality gaps, whether they modulate such changes independently from physiological factors or are mediated by them is not clear and needs additional examination.

Although there are compelling theoretical explanations for the biological foundations of a late-life reduction of the sex mortality gap, empirical evidence from large population-based studies is scant. Biomedical studies use research designs not suitable for the examination of these questions because they have different foci and are based primarily on animals or small regional clinical samples of a single sex and/or limited age ranges (13-17). Demographic studies focus on trends in aggregate rates but not explicit measures of biological robustness or social processes leading to mortality $(7,8)$. Age variations in sex differences in mortality have not been systematically examined from an integrative biological and sociodemographic perspective or rigorously modeled in population-based studies (20). It is unclear how differential exposures and vulnerabilities to social and physiological risk factors contribute to age variations in the sex mortality gap by cause. It also remains to be determined whether the mechanisms underlying sex differences are specific to the life-course stage and the cause of death. In this study, we address these questions and further test the hypothesis of a late life reduction of the sex mortality gap by directly linking social behavioral and physiological 
variables to age variations in total and cause-specific mortality risks. The results should shed more light on sex phenotypic differences throughout the life span and may lead to more effective health care designs for achieving longer and healthier lives for both sexes.

\section{METHODS}

\section{Study Population}

The data come from the National Health and Nutrition Examination Survey (NHANES) Linked Mortality Study public-use file 1988-2006. The NHANES, conducted by the National Center for Health Statistics, uses a multistage stratified sampling design and includes a representative sample of the noninstitutionalized U.S. population, with an oversample of older persons and minorities (26). The study sample of 25,254 respondents aged 17+ attended household interviews or clinical examinations in 1988-1994, 1999-2000, 2001-2002, and 2003-2004 and were eligible for mortality follow-up through 2006, the most recent year available in the public-use file. Mortality information is based on probabilistic matching between NHANES and National Death Index death certificate records. The National Center for Health Statistics matching methodology is similar to the standard methodology offered by the National Death Index, which selects death record matches on the basis of 7 established match criteria (27). Respondents who were not identified as deceased by the end of the follow-up period were assumed to be alive. The analytic sample for the follow-up period of up to 18 years recorded 1902 deaths. Table 1 summarizes the major causes of death examined. We divided malignancies into lung and other cancers in consideration of the uniquely strong link between lung cancer and smoking. We adopted the International Classification of Diseases underlying-cause-of-death recode across all years in the study period (28).

The sample consists of 13,183 women and 12,071 men in three age groups: young adulthood (17-44 years), middle age (45-64 years), and older age (65 years and older). Table 2 summarizes sample characteristics by age and sex in terms of descriptive statistics of covariates in regression analyses. These include demographic and social factors, health behaviors, 12 biomarkers of physiological dysregulation ( 2 for systemic inflammation, 8 for the metabolic syndrome $(19,29)$ and related disorders, homocysteine, and creatinine clearance), morbidity, and general health status. Laboratory measurements and assay procedures for all biomarkers have been described elsewhere $(30,31)$.

\section{Analytic Methods}

For descriptive analyses, age-specific death rates by cause were calculated as the number of deaths per 100,000 person-years; logged male to female ratios of death rates were then computed to compare sex differences. Cox proportional hazards regression models were estimated to test the statistical significance of observed sex differences and age variations therein. In light of previous research $(7,20)$, we examined three substantively meaningful age categories in final analyses because the death counts are too small to compute stable estimates of death rates and hazard ratios (HRs) for more refined age intervals and the age distributions do not differ by sex within each age group. We first estimated unadjusted HRs, or gross effects, for sex, age, and sex-by-age interaction. Adjustments of covariates were then added to test for net effects. Continuous variables were recoded into intervals for the identification of nonlinear effects and more stable estimates of HRs. We dichotomized biomarkers into high- and low-risk groups by using cutoff points based on clinical practice for 11 markers and empirically defined for creatinine clearance as the top quartile at risk based on prior studies of the same data $(20,32)$. For each control variable, a Cox model with only that variable was first estimated. The variable was allowed to enter the multivariate models if its unadjusted HR was statistically significant (the $P$ for entry was $<.05$ and for 
removal was .10). Groups of control variables were then added stepwise. The results for these models were similar to those in the full models including all variables. The same variables were assessed in models for each cause of death for comparison of risk factors across the causes and to age-specific models by cause.

We adopted the common practice of censoring survival times at competing causes of death (33), that is, when estimating the model for a particular cause, we treated all other death causes as censoring. Small percentages of respondents are missing data on most biomarkers (ranging from $1.5 \%$ to $9.5 \%$ ) that indicate unusable information, incapacity, or refusal to provide biospecimens due to old age, frailty, or additional risk factors $(30,31)$. We added a missing value category to all biomarkers in regression models. We then compared analyses that excluded and included the missing category. These two approaches did not produce substantively different results; thus, we report the results of the latter as it yields slightly more conservative findings. We used Stata 10.0 for all statistical analyses. To adjust for the complex survey design of the NHANES, we applied sampling weights for descriptive analyses and used the "svy" procedures for regression analyses.

\section{RESULTS}

The logged age-specific sex mortality ratios in Figure 1 show greater rates of male mortality for all ages and most causes except nonlung cancers, for which mortality rates are greater for females at younger ages of 17-44. Ratios decreased in the middle and older ages for total mortality and mortality attributable to circulatory and infectious diseases, pneumonia and influenza (P\&I), and external causes, indicating smaller male excesses. However, the ratios increased in the older ages for cancer-related deaths.

Unadjusted HRs of the model for total mortality in Table 3 show a significant male excess in mortality risk $(\mathrm{HR}=2.26, p<.001)$ and strong age variations $(\mathrm{HR}$ of 45-64 years $=9.18$, HR of $65+$ years $=47.11, p<.001$ ), and also a reduction of the male excess in late life (HR of sex $\times(45-64)=0.58$, HR of $\operatorname{sex} \times(65+)=0.60, p=.02)$. Adjusting for covariates largely reduced the age coefficients and slightly reduced the sex-by-age $65+$ interaction effect, indicating that social, behavioral, physiological, and morbidity factors partially account for the old-age reduction of the sex gap.

Compared with the results of total mortality, the unadjusted HRs for the circulatory disease mortality model showed a similar male excess and a greater reduction of this excess in older ages. The reduction became nonsignificant after we adjusted for social behavioral and physiological functions. Interestingly, mortality from cancer revealed no significant differences by sex when we adjusted for age and sex-by-age interaction effects. There were, however, significant age changes in sex difference that indicated a large increase in male excess in cancer mortality risk beyond 65 years of age, when most cancers occur ( $\mathrm{HR}=$ $1.87, p=.004)$. This effect remains significant and sizable after adjustment of other risk factors. The site-specific model estimates are consistent with findings on cancer incidence. Specifically, data from Surveillance, Epidemiology, and End Results show greater incidences of lung cancer and prostate cancer that occur only in men that increase sharply at older ages. Correspondingly, unadjusted HRs show increases in male excesses in lung and other cancer mortality risks after 65 years of age. The Surveillance, Epidemiology, and End Results data also show a slight female excess of cancers before age 60 as the result of breast cancer, which occurs at a greater rate earlier in life than the other cancers (34).

Similarly, there was a small female excess in nonlung cancer mortality that reverses after the age of 65 . The lack of significance in lung cancer mortality results may be attributable to the small number of deaths in this data set. Our adjustment for smoking and other covariates 
reduced the increases in male excesses in cancer mortality risks in older ages. Sex differences in infectious disease and P\&I mortality are enormous, with male subjects having much greater rates of death. Most of these sex differences occur during reproductive years and largely decline thereafter. A more active female immune system during reproductive ages could potentially explain this apparent difference and its change over the life span, as suggested by the immune competence hypothesis. Results on external causes of death do not suggest any significant age change in the sex gap, which also indirectly supports the biological hypothesis as reproductive apparatus does not have obvious bearings on exogenous forces of mortality.

Figure 2 compares the HRs of sex differences for all causes by age group, with adjustment for other factors. Consistent with the aforementioned results, the major finding is that the sex mortality gap shows strong age variations and is cause specific. For total mortality, the adjusted HRs are all greater than 1, indicating the well-known male excess. The adjusted HR for middle age, however, is not significant. Although the circulatory disease mortality result indicates consistent female advantages across all ages, the sex difference is ambiguous for cancer mortality in young and middle ages and reverses in direction during older ages. The male excess in mortality risk is not significant in either middle or older ages for remaining causes. Reductions in the sex gaps are observed in late life for total mortality and mortality caused by causes associated with physiological functions that show substantial changes with age, including circulatory diseases, infectious diseases, and P\&I. For mortality by cancer, no clear sex difference is observed before older ages, but a large male excess occurs during older ages. There are no significant age variations in the sex gaps for external and residual causes of death. Comparisons of the unadjusted and adjusted HRs in Table 3 also indicate that differential exposures to social, physiological, and morbidity risk factors account for the late life change of sex mortality gaps completely for circulatory diseases and partially or minimally for cancers and nondegenerative diseases.

We further examined measured social behavioral and biomarkers in hazards models of cause-specific mortality by age group. Table 4 (35) presents the results for total, circulatory disease, and cancer mortality (results of covariates did not show much significance for other causes and are omitted). The significance of these risk factors varies by age and cause. For instance, racial and ethnic differences in mortality are significant in younger and older ages. Education significantly affects total mortality at younger ages. Marital status strongly affects circulatory disease mortality (being widowed young is associated with a sevenfold increase in risk of death) but not cancer mortality. Cigarette smoking exerts the most consistent detrimental effects on survival across ages and causes of death and is the single most powerful risk factor for cancer mortality among all covariates examined. In most cases, moderate drinking reduces mortality risks whereas heavy drinking increases mortality risks. Such effects are larger for cancer mortality. Physical activity is protective against circulatory disease mortality but not cancer mortality in middle and older ages. High-risk levels of systemic inflammation marked by C-reactive protein and albumin are related to substantially greater risks of mortality. An excess waist circumference and obesity are associated with greater risks of cancer mortality in younger ages but lower risks of circulatory disease mortality in older ages. High diastolic blood pressure is predictive of mortality at younger ages. In sum, social status and relationship are more important risk factors for mortality in younger ages, behaviors and lifestyles are significant for all ages, and physiological dysregulation is more predictive of mortality in older ages.

\section{DISCUSSION}

This study challenges an assumption implicit in some previous research on sex differences in human mortality risk that such differences apply uniformly throughout the life span in 
direction and magnitude. Through a modeling approach that integrates data on mortality, biomarkers and social factors, this study reveals diverse patterns of the sex gaps in mortality by cause across the life span. Additional new findings emerge here regarding how established mortality risk factors affect sex differences in different periods of development and across causes of death.

The pattern of cancer mortality is opposite to the expected late life reduction of the sex mortality gap. Given the physiological pathways to intrinsic mortality shared by degenerative diseases, this difference seems an anomaly that requires further considerations. One explanation is that the convergences in the sex gap occur in changes in age slopes instead of age slopes themselves. For example, women showed slower rates of deceleration than men in cancer incidence (31) and mortality (7) in older ages. An alternative but not mutually exclusive explanation is that trends in total cancer deaths mask the heterogeneity of individual cancer sites with unique age trajectories of progression to mortality. We also note that a wide array of social and physiological factors included in the analyses do not account for sex differences in cancer mortality and their age variations. These findings likely reflect the complexity of cancer biology and sex differences in exposures and vulnerabilities to carcinogens related to both constitutional endowment and reproductive biology. In addition, the statistical power for the calculation of age change in mortality risk and sex differences is necessarily limited by the small number of deaths in the survey. As a result, we cannot estimate reliable age slopes and their accelerations by using finer-grained age intervals for any cause-specific mortality. Future studies are needed that use population-level mortality data by detailed cancer site to further model age effects with adequate statistical power.

Selective survival cannot be ruled out as an alternative mechanism producing the age changes in sex differences. Selection of the elite eliminates the frail early on so that it decreases heterogeneity in the surviving population later in life. If this were the only mechanism operating, we should have observed converging sex gaps across all causes of death. Instead, the age patterns of sex differences vary by cause of death and adjustment of covariates. Another outcome of selection is the slowing down of the age increment in mortality risks for all subgroups. We could not directly test this in the present study due to the small number of age-specific deaths, but a prior study of age acceleration of physiological risks of mortality, such as metabolic syndrome and allostatic load, shows no similar leveling in age patterns in men and women (20). While extant evidence seems not entirely consistent with the selection hypothesis, differential selection processes by sex remain a possibility for future studies.

\section{Acknowledgments}

We thank Kathleen Mullan Harris, Dana Glei, and Jennifer Dowd, in addition to anonymous reviewers, for helpful comments.

Supported by National Institute of Aging grant number K01AG036745, awarded to the first author, and University Cancer Research Funds at the Lineberger Cancer Center, University of North Carolina at Chapel Hill.

\section{Selected Abbreviations and Acronyms}

$\begin{array}{ll}\text { NHANES } & \text { National Health and Nutrition Examination Survey } \\ \text { HR } & \text { hazard ratio } \\ \text { P\&I } & \text { pneumonia and influenza }\end{array}$




\section{REFERENCES}

1. Brody, JA.; Brock, DB. Epidemiologic and Statistical Characteristics of the United States Elderly Population. In: Finch, CE.; Schneider, EL., editors. Handbook of the Biology of Aging. 2nd ed. Van Nostrand Reinhold; New York: 1985. p. 3-26.

2. Finch, CE. The Biology of Human Longevity: Inflammation, Nutrition, and Aging in the Evolution of Life Spans. Elsevier; Amsterdam: 2007.

3. Austad SN. Why women live longer than men: Sex differences in longevity. Gender Med. 2006; 3:79-92.

4. Weden MM, Brown RA. Historical and life course timing of the male mortality disadvantage in Europe. Biodemography Soc Biol. 2008; 53:61-79.

5. Institute of Medicine. Exploring the Biological Contributions to Human Health: Does Sex Matter?. National Academy Press; Washington, DC: 2001.

6. Carey JR, Liedo P, Orozco D, Tatar M, Vaupel JW. A male-female longevity paradox in medfly cohorts. J Animal Ecol. 1995; 64:107-116.

7. Horiuchi S. Postmenopausal acceleration of age-related mortality increase. J Gerontol A Biol Sci Med Sci. 1997; 52A:B78-B92. [PubMed: 9008661]

8. Yang Y. Trends in U.S. adult chronic disease mortality: Age, period, and cohort variations. Demography. 2008; 45:387-416. [PubMed: 18613487]

9. Travato F, Lalu NM. Contribution of cause-specific mortality to changing sex differences in life expectancy: Seven Nations case study. Soc Biol. 1998; 45:1-20. [PubMed: 9584575]

10. Wingard, DL.; Cohn, BA. Variations in Disease-Specific Sex Morbidity and Mortality Ratios in the United States. In: Ory, MG.; Warner, HR., editors. Gender, Health, and Longevity: Multidisciplinary Perspectives. Springer; New York: 1990. p. 25-37.

11. Medawar, PB. Unsolved Problems of Biology. H. K. Lewis; London: 1952.

12. Kirkwood TBL. Evolution of aging. Nature. 1977; 270:301-304. [PubMed: 593350]

13. Eskes T, Haanen C. Why do women live longer than men? Eur J of Obstet Gynecol Reprod Biol. 2007; 133:126-133. [PubMed: 17324494]

14. Schuurs AHWM, Verheul HAM. Effects of gender and sex steroids on the immune response. J Steroid Biochem Mol Biol. 1990; 35:157-172.

15. Da Silva JA. Sex hormones and glucocorticoids: interactions with the immune system. Ann NY Acad Sci. 1999; 876:102-117. [PubMed: 10415599]

16. Goran MI. Energy metabolism and obesity. Med Clin North Am. 2000; 84:347-362. [PubMed: 10793646]

17. Barrett-Connor E. Sex differences in coronary heart disease: Why are women so superior? The 1995 Ancel Keys lecture. Circulation. 1997; 95:252-264. [PubMed: 8994444]

18. Fried LP, Kronmal RA, Newman AB, et al. Risk factors for 5-year mortality in older adults: the Cardiovascular Health Study. JAMA. 1998; 279:585-592. [PubMed: 9486752]

19. Lakka H, Laaksonen DE, Lakka TA. The metabolic syndrome and total and cardiovascular disease mortality in middle-aged men. JAMA. 2002; 288:2709-2716. [PubMed: 12460094]

20. Yang Y, Kozloski M. Sex differences in age trajectories of physiological dys-regulation: Inflammation, metabolic syndrome, and allostatic load. J Gerontol A Biol Sci Med Sci. 2011; 66A: 493-500. [PubMed: 21350248]

21. Waldron, I. Gender Differences in Mortality - Causes and Variation in Different Societies. In: Conrad, P., editor. The Sociology of Health and Illness: Critical Perspectives. 7th ed. Worth Publishers; New York: 2004. p. 38-55.

22. Pampel F. Cigarette use and the narrowing sex differential in mortality. Population Dev Rev. 2002; 28:77-104.

23. Verbrugge LM. The twain meet: Empirical explanations of sex differences in health and mortality. J Health Soc Behav. 1989; 30:282-304. [PubMed: 2778300]

24. House JS, Lepkowski JM, Kinney AM, et al. The social stratification of aging and health. J Health Soc Behav. 1994; 35:213-234. [PubMed: 7983335] 
25. Yang Y, Lee LC. Sex and race disparities in health: Cohort variations in life course patterns. Social Forces. 2009; 87:2093-2124.

26. Centers for Disease Control and Prevention. [Accessed July 1, 2009] National Health and Nutrition Examination Survey. Available at: http://www.cdc.gov/nchs/nhanes. htm.

27. National Center for Health Statistics. Office of Analysis and Epidemiology, The Third National Health and Nutrition Examination Survey (NHANES III) Linked Mortality File, Mortality followup through 2006: Matching Methodology. Hyattsville, MD: May. 2009 Available at: http://www.cdc.gov/nchs/data/datalinkage/matching_methodology_nhanes3_final.pdf.

28. Wheatcroft, G.; Cox, CS.; Lochner, KA. Comparative Analysis of the NHANES III Public-Use and Restricted-Use Linked Mortality Files. National Center for Health Statistics; Hyattsville, MD: 2007.

29. National Institutes of Health. Third Report of the National Cholesterol Education Program Expert Panel on Detection, Evaluation, and Treatment of High Blood Cholesterol in Adults (Adult Treatment Panel III). National Institutes of Health; Bethesda, MD: 2001.

30. Centers for Disease Control and Prevention. [Accessed July 1, 2009] Laboratory Procedures Used for the Third National Health and Nutrition Examination Survey (NHANES III), 1988-1994. 1996. Available at: http://www.cdc.gov/nchs/data/nhanes/nhanes3/cdrom/nchs/manuals/labman.pdf.

31. Centers for Disease Control and Prevention. [Accessed July 1, 2009] NHANES IV Laboratory Methods, 1999-2000. http://www.cdc.gov/nchs/nhanes/nhanes1999-2000/lab99_00.htm.

32. Crimmins EM, Johnston M, Hayward M, et al. Age differences in allostatic load: an indexof physiological dysregulation. Exp Gerontol. 2003; 38:731-734. [PubMed: 12855278]

33. Allison, PD. Survival Analysis Using SAS: A Practical Guide. 2nd ed. SAS Institute; Cary, NC: 2010.

34. Frank, SA. Dynamics of Cancer: Incidence, Inheritance, and Evolution. Princeton University Press; Princeton, NJ: 2007.

35. Boronat M, Saavedra P, Varillas VF, et al. Differences in traditional and emerging cardiovascular risk factors of subjects discordantly classified by metabolic syndrome definitions of the International Diabetes Federation and the National Cholesterol Education Program. Nutr Metab Cardiovasc Dis. 2009; 19:417-422. [PubMed: 18819785] 

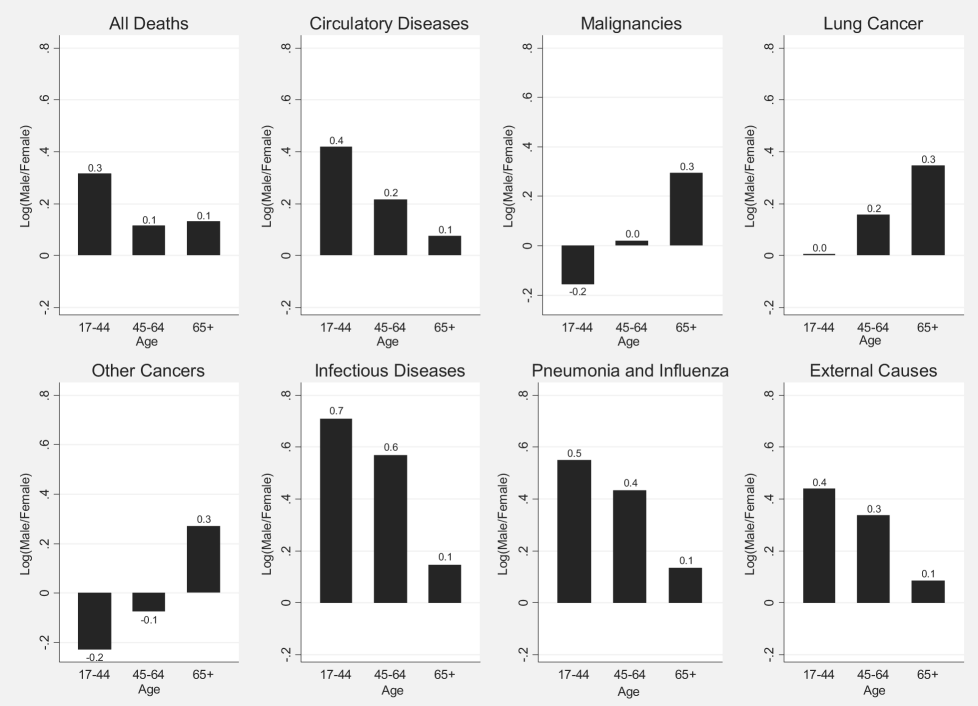

FIGURE 1.

Logged Ratio (Male/Female) of Age-Specific Mortality Rate (Weighted) by Cause of Death*

*Because the size of the sex ratio will depend on the choice of numerator, taking the log of the ratio makes it symmetrical (3). Positive values of the logged ratio indicate higher male mortality rates relative to female mortality rates; negative values indicate lower male relative to female rates. 

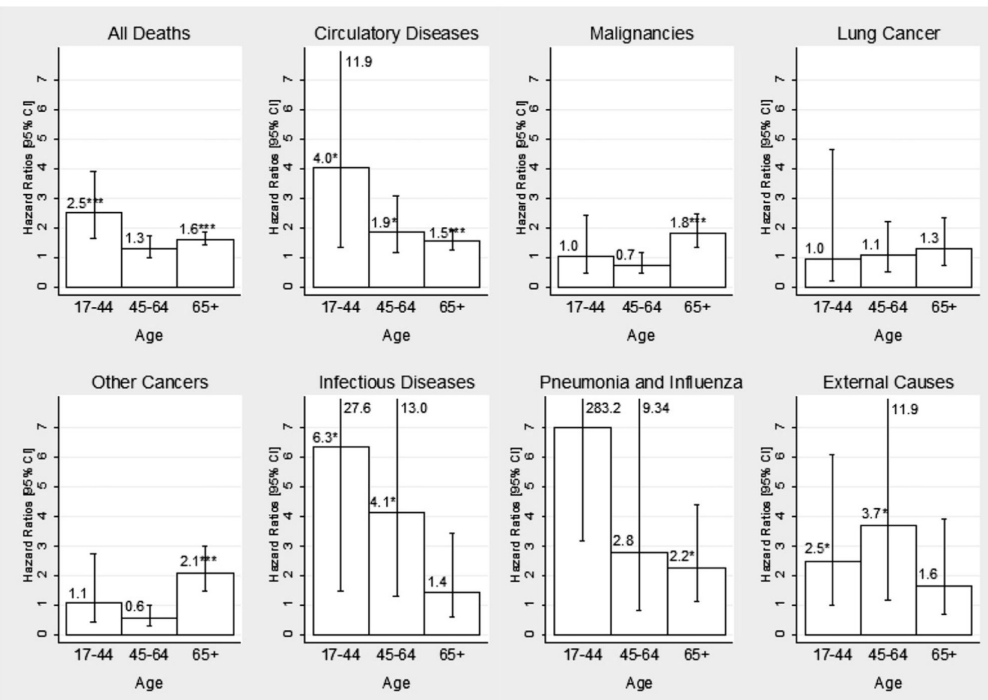

FIGURE 2.

Adjusted Hazard Ratios for Sex Difference by Age Group and Cause of Death. $* p<.05, * * p<.01, * * * p<.001 ; 2$-sided test. For P\&I, ages 17-44, the HR is 29.9 ( $p=$. 003). Numerical values of upper bounds of CIs are given when too large to include in the graph. 
TABLE 1

Causes of death coding and summary statistics (weighted): NHANES 1988-2006 mortality follow-up study analytic sample $(N=25,254$; average follow-up $=9.2$ years $)$

\begin{tabular}{llrrc}
\hline Cause of death & ICD-10 codes & No. deaths & Death rate (per 100,000 person-years) & Age at death, mean (SD) \\
\hline All causes & & 1902 & 1144.60 & $73.35(14.65)$ \\
Circulatory diseases ${ }^{*}$ & 100-178, 180-199 & 724 & 435.69 & $76.76(13.08)$ \\
Malignant neoplasms & C00-C97 & 494 & 297.28 & $69.98(13.11)$ \\
$\quad$ Lung cancer & C33-C34 & 177 & 106.52 & $68.46(11.82)$ \\
$\quad$ Other cancers & C00-C32, C35-C97 & 318 & 191.37 & $70.82(13.71)$ \\
Infectious and parasitic diseases & A00-B99 & 68 & 40.92 & $64.09(18.29)$ \\
Pneumonia and influenza & J10-J18 & 51 & 30.69 & $80.54(11.65)$ \\
External causes & V01-Y88 & 88 & 52.96 & $57.58(20.33)$ \\
Other causes & Residuals & 478 & 287.65 & $75.12(13.61)$ \\
\hline
\end{tabular}

ICD = International Classification of Diseases; NHANES = National Health and Nutrition Examination Survey.

We separately examined cardiovascular and cerebrovascular diseases as subcategories of the circulatory diseases in additional analyses but only report results of the latter because of a lack of significant differences in subsequent analyses. 


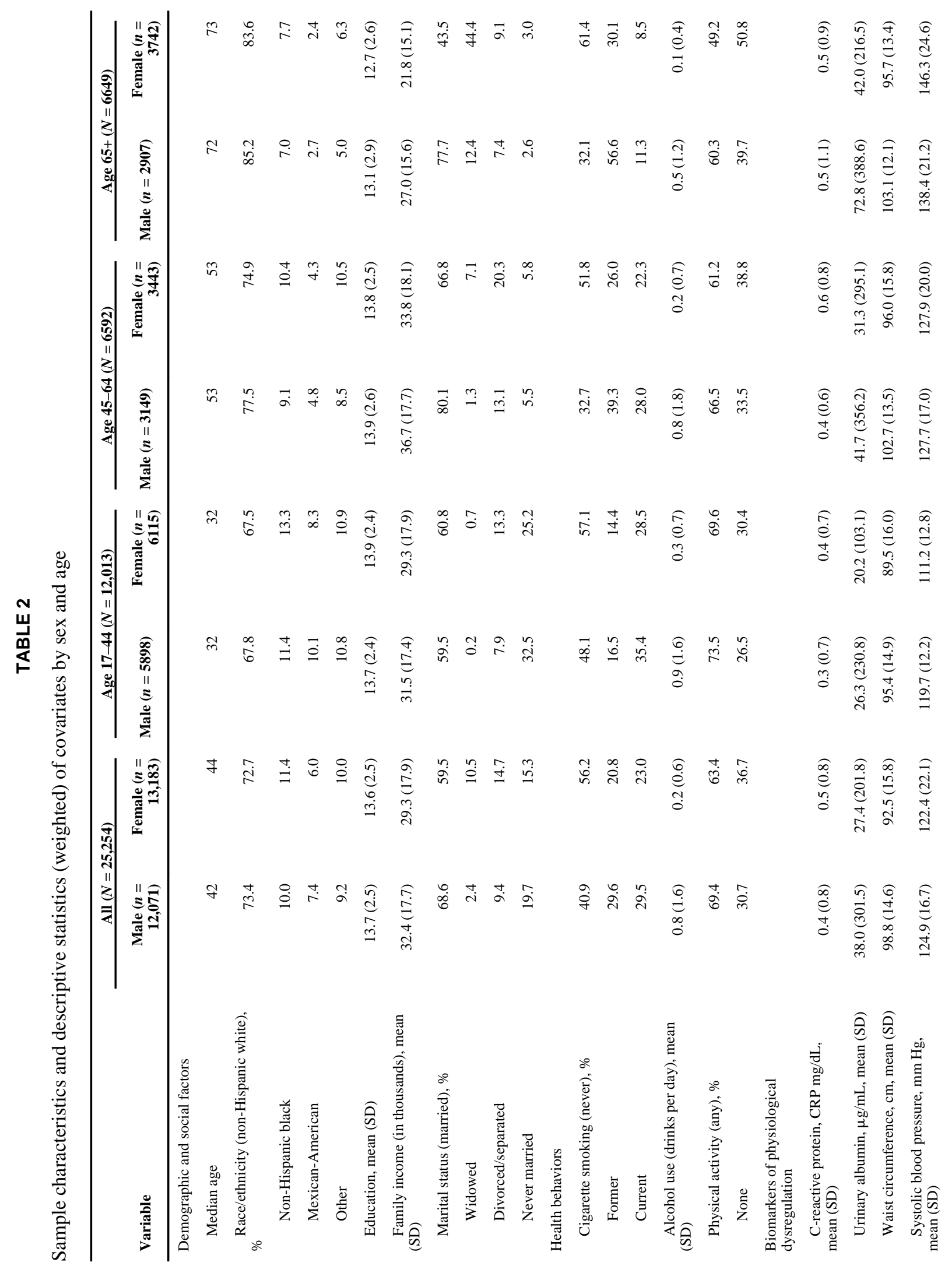




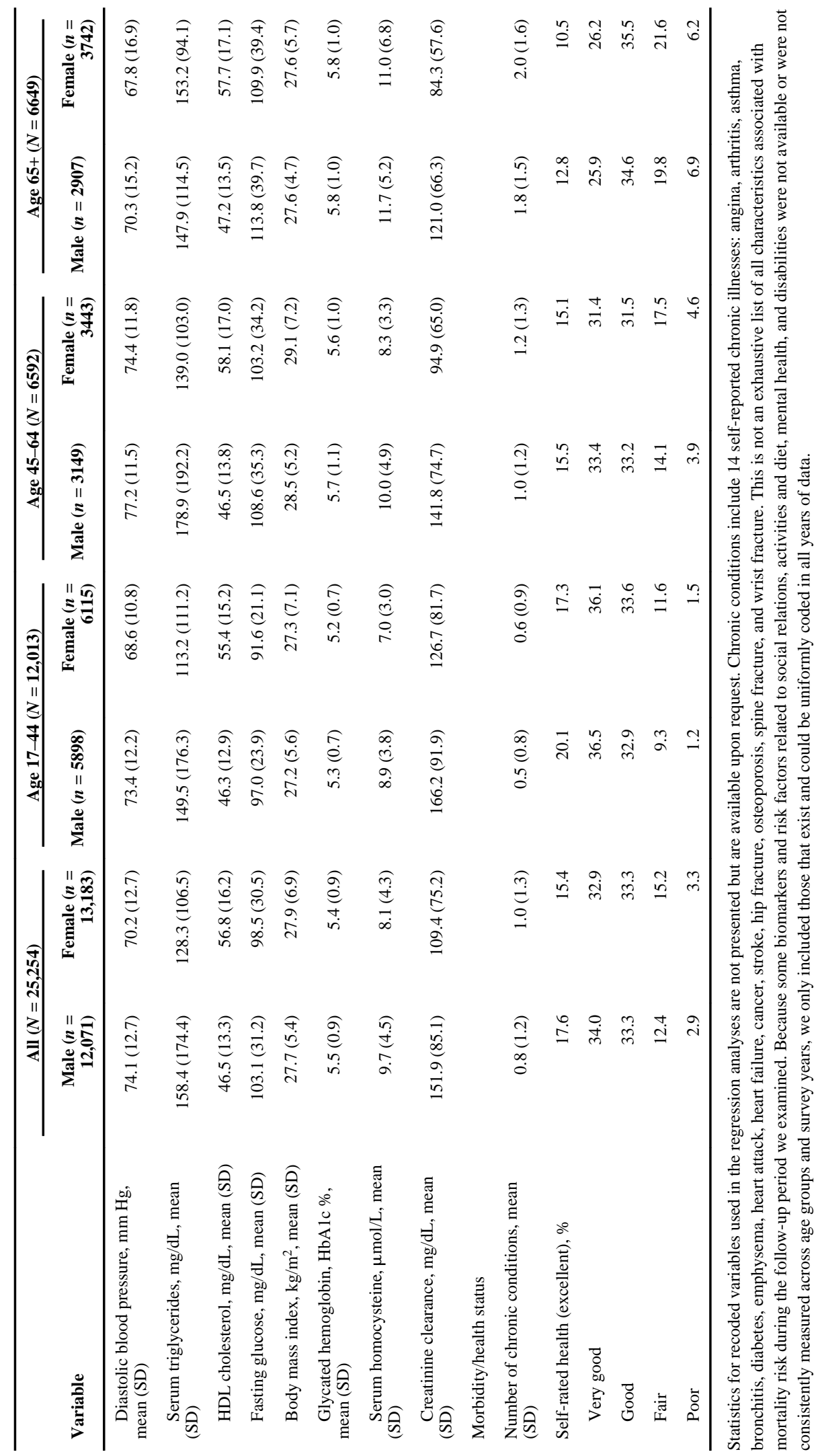




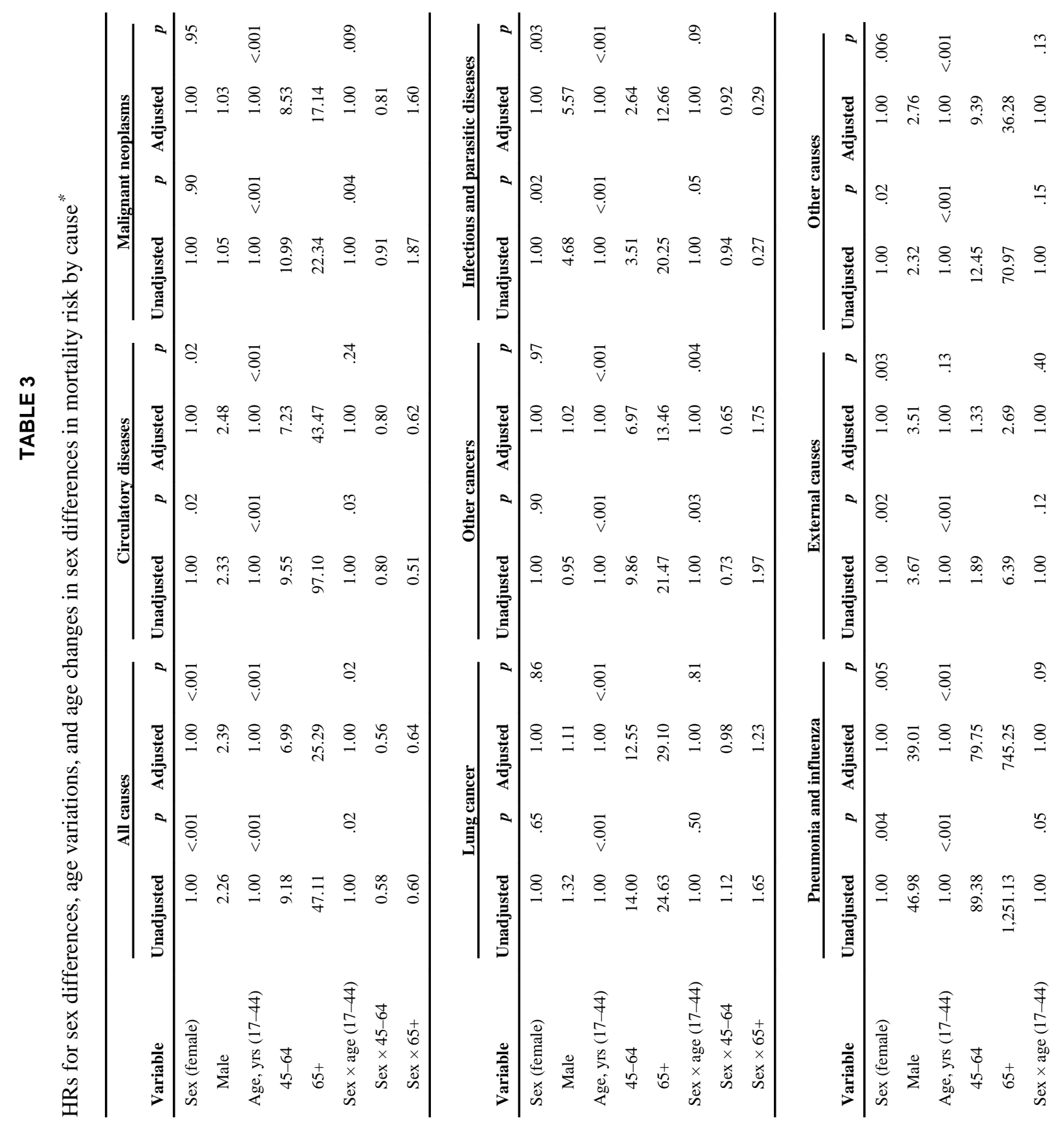




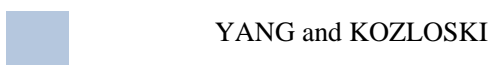

Page 15

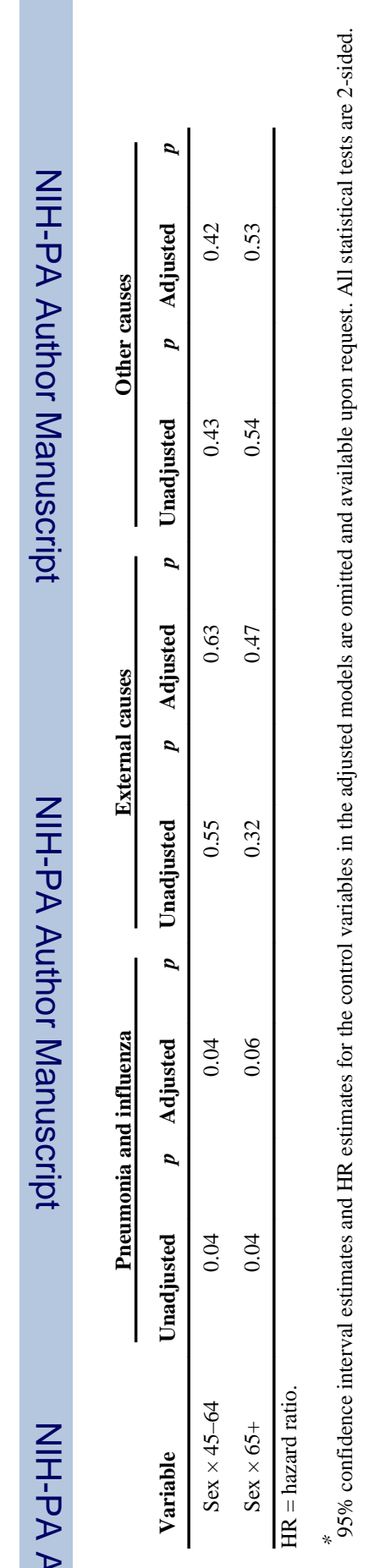

을

Ann Epidemiol. Author manuscript; available in PMC 2013 February 01. 


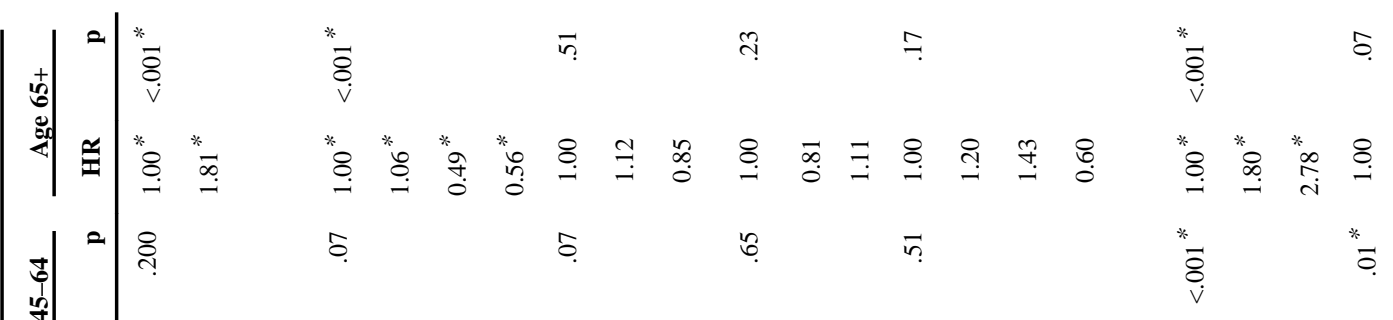

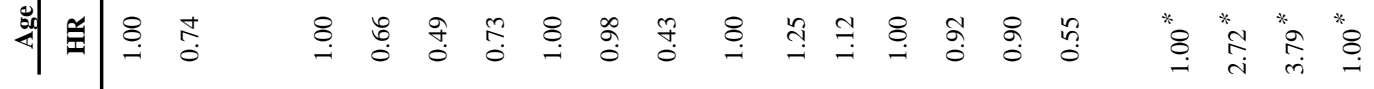

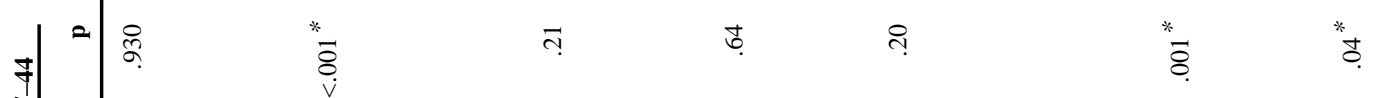

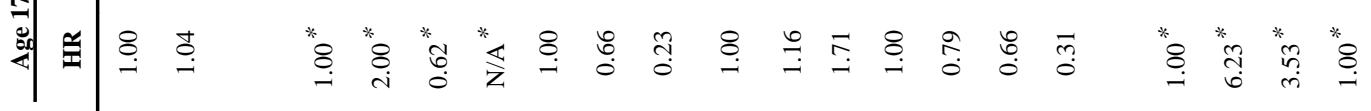

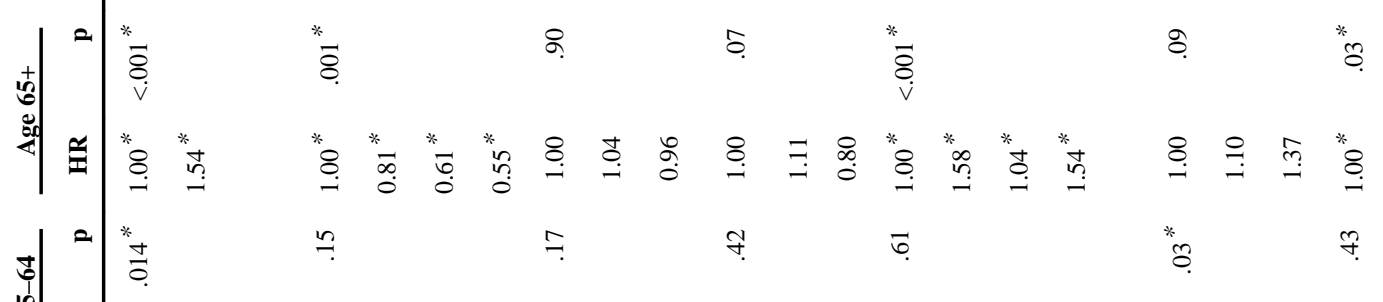

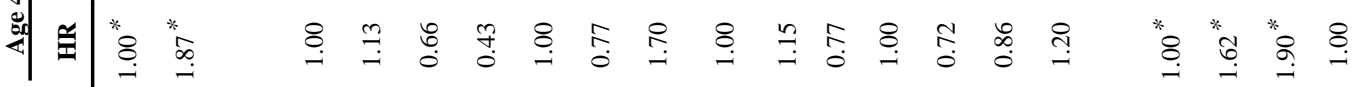

$\stackrel{5}{\circ} \quad$ *

*

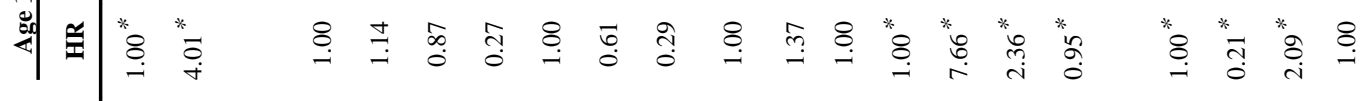

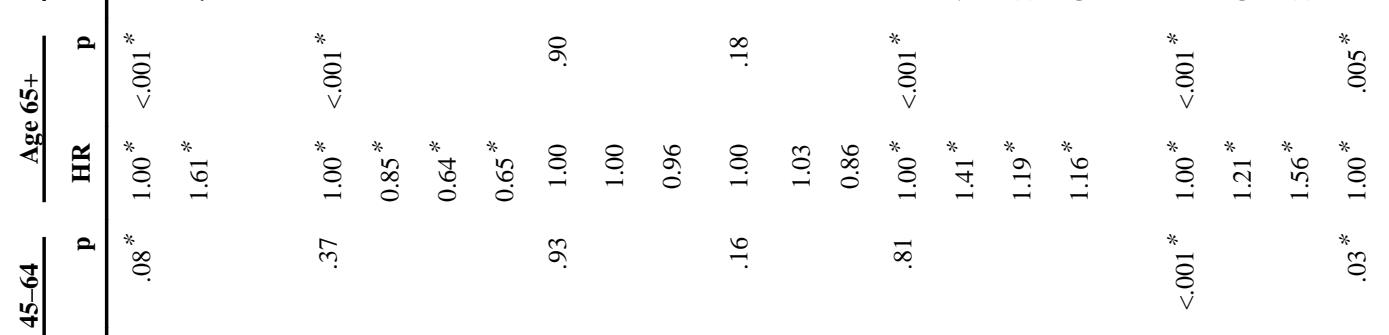

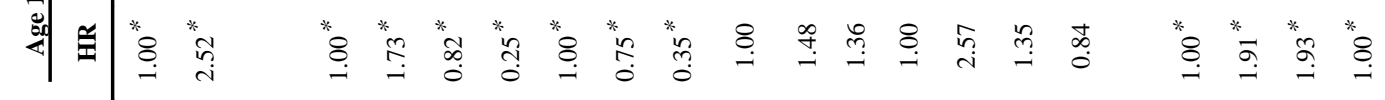




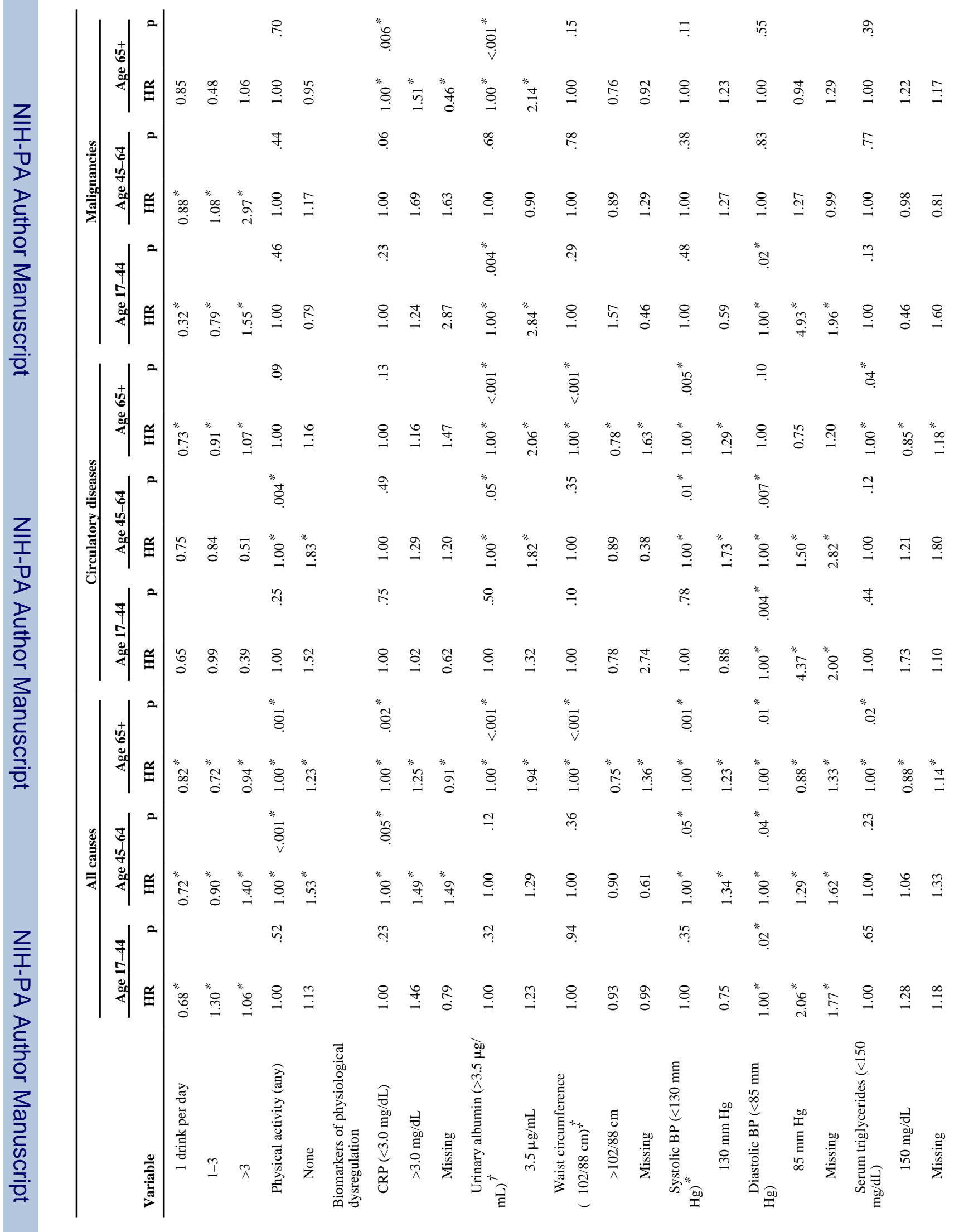




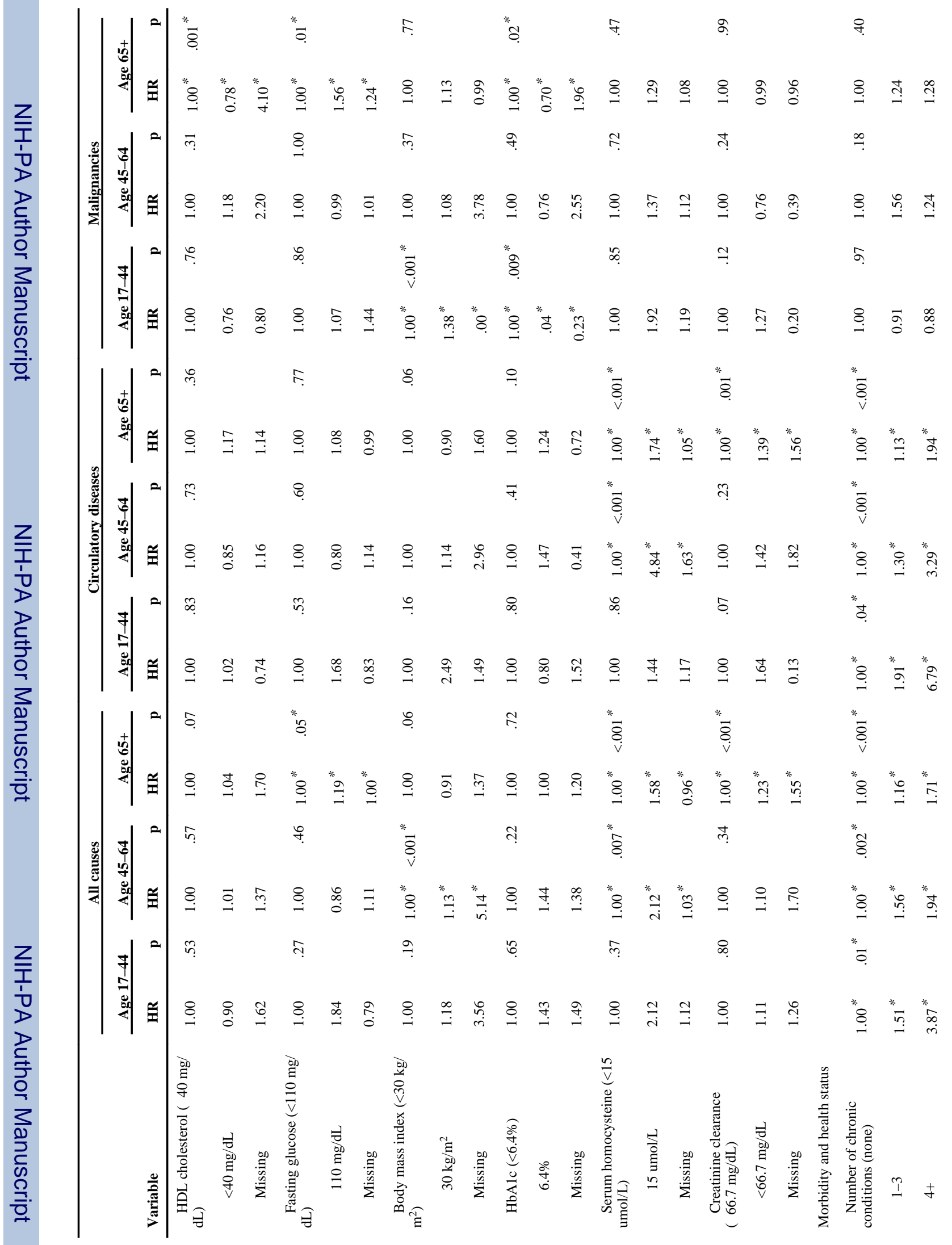




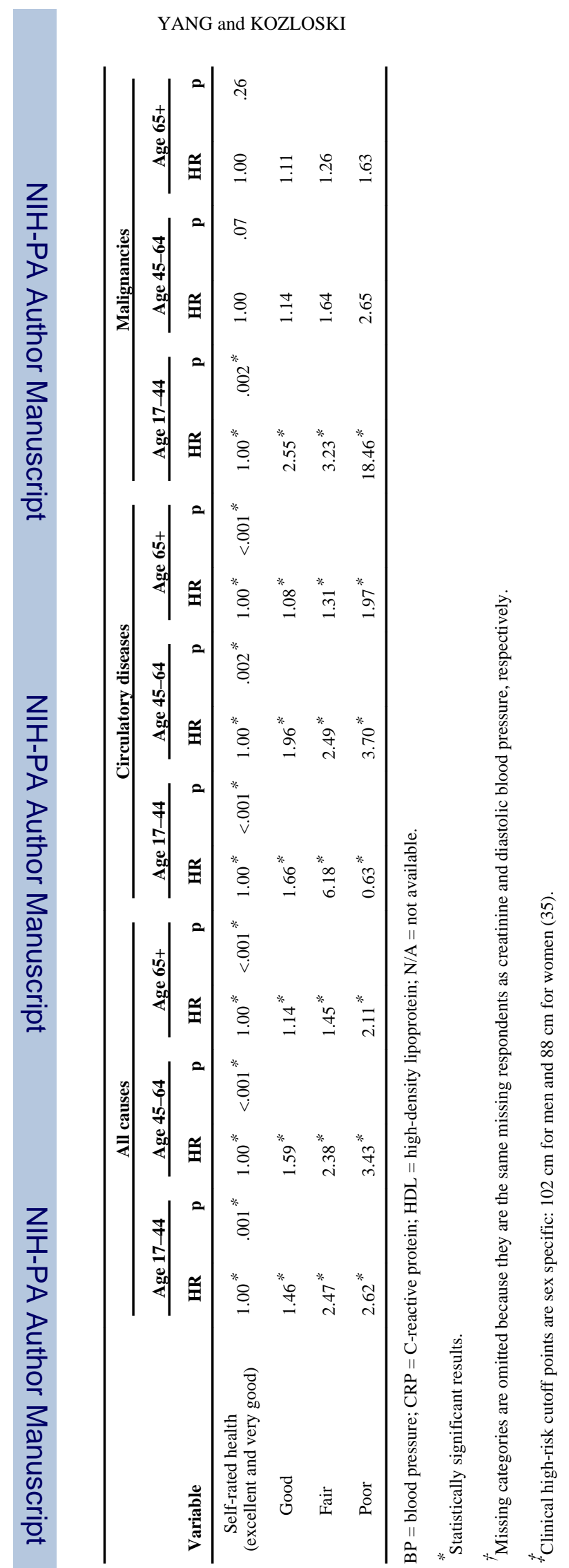

Page 19 